\title{
Veterans with Hepatitis B, who is Being Missed and Why?
}

\author{
We'am Hussain ${ }^{1 *}$, Patrick Chen ${ }^{2}$, and Padmini Krishnamurthy ${ }^{3}$ \\ ${ }^{1}$ Resident Wright State University, Boonshoft School of Medicine Department of Internal Medicine,USA \\ ${ }^{2}$ Gastroenterology Fellow Wright State University, Boonshoft School of Medicine, Department of Internal Medicine, Division of Gastroenterology, USA \\ ${ }^{3}$ Gastroenterology, Wright State University, Boonshoft School of Medicine Department of Internal Medicine, Division of Gastroenterology, USA
}

*Corresponding author: We'am Hussain, Resident Wright State University, Boonshoft School of Medicine Department of Internal Medicine, USA, E-mail: Hussain.Weam@gmail.com

Received: 20 Jul, 2020 | Accepted: 19 Aug, 2020 | Published: 25 Aug, 2020

Citation: Hussain W, Chen P, Krishnamurthy P (2020) Veterans with Hepatitis B, who is Being Missed and Why? Clin Res Open Access 6(2): dx.doi. org/10.16966/2469-6714.158

Copyright: $(2020$ Hussain W, et al. This is an open-access article distributed under the terms of the Creative Commons Attribution License, which permits unrestricted use, distribution, and reproduction in any medium, provided the original author and source are credited.

\section{Abstract}

Background and Aim: On May 23, 2018 the Veterans Administration (VA) issued a directive to establish policies for improving prevention, early diagnosis, and treatment for all viral hepatitis including Chronic Hepatitis B (CHB). The goals of our study were to identify patients with a positive HBsAg test at the Dayton VA Medical Center who would benefit from medical care for CHB with our gastroenterology (GI) clinic, identify reasons for lack of treatment, and re-establish patients into the GI clinic.

Methods: We reviewed patients with a diagnosis of Chronic Hepatitis B using ICD-9 and ICD-10 codes who were receiving care at the Dayton VA Medical Center through May 23, 2018.

Data were gathered retrospectively for demographics, clinical labs, imaging, and presence of cirrhosis, treatment status, and hepatocellular carcinoma surveillance. Following American Association for the Study of Liver Diseases guidelines for Hepatitis B, we used patient data to identify those who would benefit from further GI follow-up.

Results: A total of 61 patients had a diagnosis of $\mathrm{CHB}, 11$ with cirrhosis and 50 without cirrhosis.

Among patients with cirrhosis, four were not on treatment (two noncompliant with follow-up, one with severe comorbidities, one lack of provider knowledge). For patients without cirrhosis, $43 / 50$ were not on treatment. Of these $43,16 / 43(37.2 \%)$ patients did not have an indication for treatment. For the remaining 27/43 patients: 22/43 (51.2\%) were non-compliant with follow-up visits, 4/43 (9.3\%) had severe comorbidities, and $1 / 43(2.3 \%)$ was not treated due to lack of provider knowledge.

Excluding patients with severe comorbidities, loss of surface antigen, normal serum alanine aminotransferase (ALT), or low viral load, 26 patients ( 3 with cirrhosis and 23 without cirrhosis) would benefit from follow-up with a GI provider. We mailed letters to these patients encouraging them to follow up with the GI department at our facility. One-half of these patients (13/26) responded, with 11/13 (85\%) agreeing to re-establish care in the $\mathrm{Gl}$ clinic and 2/13 (15\%) declining. We contacted the primary care provider for the 13 patients who did not respond, requesting patients to be referred to $\mathrm{Gl}$ after a discussion with their patients.

Conclusion: We were successful in re-establishing $50 \%$ of patients with $\mathrm{HBsAg}$ back with our $\mathrm{GI}$ providers through enhanced identification procedures, direct contact with patients, and collaboration with other health care providers. This study can be the framework for future efforts to improve screening, diagnosis, treatment, and surveillance of patients affected by $\mathrm{CHB}$.

Keywords: Hepatitis B; Hepatocellular Carcinoma; Screening

\section{Introduction}

A positive Hepatitis B surface antigen ( $\mathrm{HBsAg}$ ) test establishes the diagnosis of Hepatitis B. Persistence of HBsAg six months to one year after an acute infection leads to chronic inactive carrier state or chronic active infection. The prevalence of HBsAg varies greatly across countries; in developed countries, the prevalence is higher among those who emigrated from high- or intermediate-prevalence countries and in those with high-risk behaviors (intravenous drug use, men who have sex with men, inmates of correctional facilities). Hepatitis B virus (HBV) is transmitted by perinatal, percutaneous, sexual exposure, or close contact with bodily fluids of those infected with HBV. HBV has been linked with Hepatocellular Carcinoma (HCC) due to integration of HBV DNA into host hepatic cells and occurs in those with a long-standing history of infection.

Hepatocellular carcinoma accounts for $85-90 \%$ of all primary liver cancers [1]. The highest HCC incidence rates are reported from 
regions endemic for Hepatitis B virus infection, including Southeast Asia and sub-Saharan Africa [1]. The incidence of HCC in inactive HBV carriers with no evidence of liver cirrhosis is less than $0.3 \%$ per year [2] versus $3.1 \%$ [3] in those with cirrhosis.

For patients with increased risk, the American Association of the Study of Liver Diseases (AASLD) guidelines recommends HCC surveillance. Imaging modalities including ultrasound, and in some cases, computed tomography or magnetic resonance imaging, is recommended to be performed every six months [4].

Under the Veterans Administration (VA) directive issued on May 23, 2018, policies to improve prevention, early diagnosis, and treatment were established for all viral hepatitis including Chronic Hepatitis B (CHB). Screening rates for HBV in the VA is $28.5 \%$ [4]. The Centers for Disease Control and Prevention's National Health and Nutrition Examination Survey (NHANES) reported that among those screened, prevalence of HBV infection is $1 \%$ compared to 0.27 $0.50 \%$ in the general population [5]. Among U.S. veterans the rate of HBV infection varies by race: Asians 6.1\%, African Americans 1.8\%, Caucasians 0.7\%, and Hispanics 0.6\% [6].

The goals of our study were to (1) identify all patients with a positive Hepatitis B surface antigen test at the Dayton Veterans Affairs Medical Center (DVAMC); (2) re-establish these patients in the GI clinic; (3) treat eligible patients according to AASLD guidelines; and (4) ensure ongoing HCC screening for at-risk patients per AASLD guidelines.

\section{Methods}

Patients with a diagnosis of Chronic Hepatitis B (CHB) using ICD9 and ICD-10 codes who were receiving care at the Dayton VAMC through May 23, 2018 were included in the study. Demographic, clinical, and lab data were gathered from the electronic medical records.

Serum Alanine Aminotransferase (ALT), Hepatitis E antigen status, Hepatitis E antibody status, and presence of cirrhosis were documented. Other clinical data gathered were follow-up with a gastroenterology provider, treatment status, reason for no treatment, and last date of HCC surveillance.

\section{Results}

Through May 23, 2018, 2180 unique patients were screened for Hepatitis B at our center. Sixty-one $(2.8 \%)$ patients carried the diagnosis of Chronic Hepatitis B, 11 (18\%) of whom had cirrhosis and $50(82 \%)$ had no cirrhosis. Table 1 describes patient characteristics. Among the 11 patients with cirrhosis, 7 (64\%) were on treatment, and $4(36 \%)$ were not on treatment.

Reasons for no treatment were failure to follow up ( 2 patients), severe comorbidities (1 patient), and lack of provider knowledge (1 patient).

Among patients without cirrhosis, 7/50 (14\%) were on Hepatitis B treatment while $43 / 50$ (86\%) were not on treatment. Of the 43 patients not on treatment, $6 / 43$ (14\%) had spontaneously cleared the HBsAg, 10/43 (23\%) did not meet the ALT or viral load criteria for treatment, 22/43 (51.2\%) were non-compliant with follow-up visits, 4/43 (9.3\%) had severe comorbidities, and $1 / 43$ (2.3\%) was not treated due to lack of provider knowledge (Table 2).

After reviewing individual patient records, we identified 26 (42.6\%), 3 with cirrhosis and 23 without cirrhosis, who would potentially benefit from follow-up by a gastroenterology (GI) provider or intervention for Hepatitis B. We mailed letters to these 26 patients encouraging them to
Table 1: Characteristics of 61 patients with diagnosis of Chronic Hepatitis B.

\begin{tabular}{|l|c|}
\hline Characteristic $[\mathbf{n}(\%)]$ & $9(14.8)$ \\
\hline Less than age 50 & $52(85.2)$ \\
\hline Ag 50 or older & $57(93.4)$ \\
\hline Sex & $4(6.6)$ \\
\hline Male & \\
\hline Female & $49(80.3)$ \\
\hline Race & $10(16.4)$ \\
\hline Caucasian & $2(3.3)$ \\
\hline African American & $7(63.6)$ \\
\hline Asian & $4(36.4)$ \\
\hline Cirrhosis & \\
\hline On treatment & $43(86)$ \\
\hline Not on treatment & $7(14)$ \\
\hline No Cirrhosis & \\
\hline On treatment & \\
\hline Not on treatment &
\end{tabular}

follow up with the GI department at our facility. Thirteen of 26 (50\%) responded, with 11 (85\%) agreeing to re-establish care in the GI clinic, and two (15\%) declining. We contacted the primary care providers of the 13 patients who did not respond to our letter asking them to refer to GI after discussion with their patients.

Regarding HCC screening, 40 (65.6\%) patients met the AASLD criteria for screening and 21 did not. Of the 40 patients who met the criteria for HCC screening, 15/40 (37.5\%) had regular screening ultrasounds, 24/40 (60\%) (22 non-cirrhotic and 2 cirrhotic) had ultrasound or CT abdomen imaging within last 6 months for other medical reasons, and 1/40 (2\%) did not have any imaging. Thus, the compliance rate for HCC screening was $37.5 \%$ (Table 3).

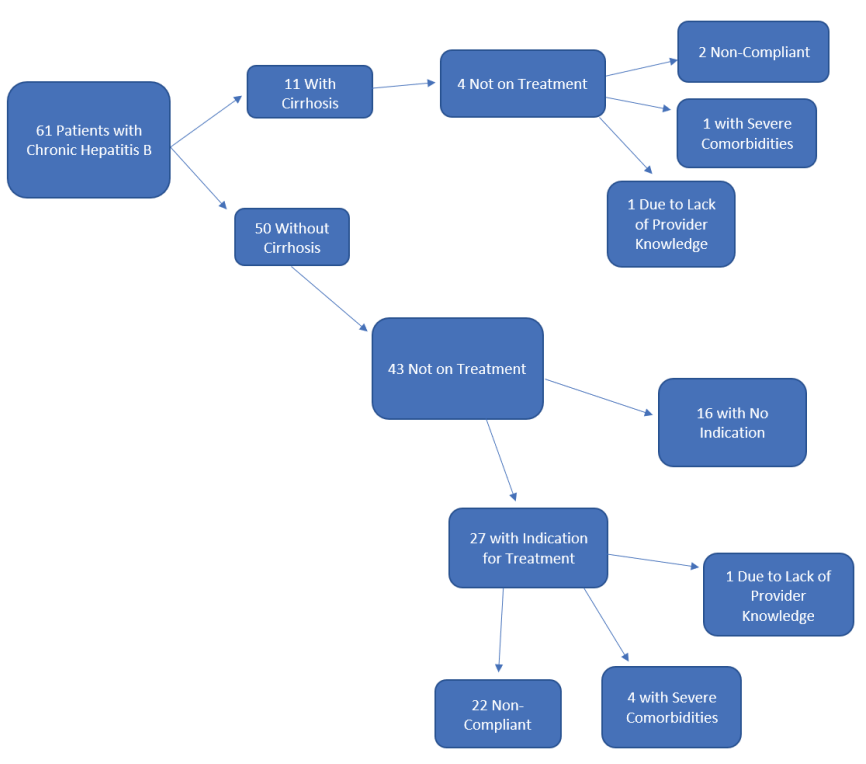


Table 2: Reasons for lack of treatment for 47 eligible patients with Chronic Hepatitis B.

\begin{tabular}{|l|c|c|}
\hline Reason [n (\%)] & $\begin{array}{c}\text { Cirrhosis } \\
(\mathbf{n}=\mathbf{4})\end{array}$ & $\begin{array}{c}\text { No Cirrhosis } \\
\text { (n=33) }\end{array}$ \\
\hline Loss of surface antigen & $0(0.0)$ & $6(14.0)$ \\
\hline Severe comorbidities & $1(25.0)$ & $4(9.3)$ \\
\hline Non-compliant with follow-up visits & $2(50.0)$ & $22(51.2)$ \\
\hline Did not meet ALT or VL criteria & $0(0.0)$ & $10(23.3)$ \\
\hline Lack of provider knowledge & $1(25.0)$ & $1(2.3)$ \\
\hline
\end{tabular}

Table 3: Patient Results.

\begin{tabular}{|l|c|c|}
\hline & Cirrhosis & Without Cirrhosis \\
\hline On Treatment & 7 & 7 \\
\hline Not on Treatment & 4 & 43 \\
\hline Non Compliance & $2(50)$ & $22(51.2)$ \\
\hline Severe Comorbidities & $1(25)$ & $4(9.3)$ \\
\hline Lack of Provider Knowledge & $1(25)$ & $1(2.3)$ \\
\hline No Indication for Treatment & 0 & $16(37.2)$ \\
\hline
\end{tabular}

\section{Discussion}

The national VA data base generates a local case registry of patients with a diagnosis of Chronic Hepatitis B for each VA medical center. The registry allows providers to identify, monitor, and track care of these patients over time [7]. Local use of the case registry and proactive measures taken nationwide by the VA's HIV, Hepatitis and Related Conditions (HHRC) program have helped to increase screening rates for HBV [6]. The aim of our study was to identify patients with positive HBsAg at our center and re-establish care for those who were lost to follow-up, back with our GI providers. We found that $42.6 \%$ of our patients with a positive Hep B surface antigen test required follow-up on a regular basis with a GI provider. In addition, compliance the rate for regular HCC screening was only $37.5 \%$. We focused on contacting these patients, educating them, and inviting them to re-establish for further management. Letters via US mail brought a $50 \%$ response rate, and most respondents were re-established in the GI clinic. Our findings are consistent with Ma et al., who reported that patient contact and education were the most effective methods in gaining acceptance for Hepatitis B intervention [8].

Poor patient compliance is a major barrier to caring for those with Chronic Hepatitis B. Lack of awareness of positive HBsAg status among affected veterans, limited knowledge of Chronic Hepatitis B's consequences, logistic barriers in keeping clinic visits (e.g., travel arrangements or distances), denial of diagnosis by the veterans, and underlying psychological or social issues all may contribute to unsatisfactory patient compliance. In addition, inadequate awareness among primary care providers may play a role in patients not being referred to GI providers. Physician leadership, education of patients, increased awareness among primary care providers, establishing an electronic reminder system, and travel/navigation services can improve compliance $[7,8]$.

\section{Conclusion}

Although the prevalence of Hepatitis B, either as inactive carrier state or chronic infection, is low in the U.S. veteran population, HBV is twice as high as in the general population. Inadequate education or treatment and lack of screening ultrasounds may result in transmission of infection to others, liver cirrhosis with decompensation, and increased risk of diagnosing HCC at late stages. We were successful in re-establishing $50 \%$ of eligible patients with positive HBsAg status back into the care of our GI providers through identification of patients with positive surface antigen, direct contact by mail, and education. This study can be a framework for future efforts to improve screening, diagnosis, treatment, and surveillance of patients affected by HBV.

\section{References}

1. El-Serag HB, Rudolph KL (2007) Hepatocellular carcinoma: epidemiology and molecular carcinogenesis. Gastroenterology 132: 2557-2576.

2. El-Serag HB (2012) Epidemiology of viral hepatitis and hepatocellular carcinoma. Gastroenterology 142: 1264-1273.

3. Thiele M, Gluud LL, Fialla AD, Dahl EK, Krag A (2014) Large variations in risk of hepatocellular carcinoma and mortality in treatment naive hepatitis B patients: systematic review with meta-analyses. PLoS One 9: e107177.

4. Marrero JA, Kulik LM, Sirlin CB, Zhu AX, Finn RS, et al. (2018) Diagnosis, Staging, and Management of Hepatocellular Carcinoma: 2018 Practice Guidance by the American Association for the Study of Liver Diseases. Hepatology 68: 723-750.

5. Yang Y, Luk J, Sofair A (2018) Epidemiology of Chronic Liver Disease in the United States. Clinical Epidemiology of Chronic Liver Diseases 10: 57-74.

6. Backus L, Belperio P, Loomis T (2013) $64^{\text {th }}$ Annual Meeting of AASLD. Hepatology 58: 208-355.

7. Sun WC, Hsu P, Yu H, Lin KH, Tsay F, et al. (2015) The Compliance of Doctors with Viral Hepatitis B Screening and Antiviral Prophylaxis in Cancer Patients Receiving Cytotoxic Chemotherapy Using a HospitalBased Screening Reminder System. PloS One 10: e0116978.

8. Ma GX, Fang CY, Seals B, Feng Z, Tan Y, et al. (2017) A CommunityBased Randomized Trial of Hepatitis B Screening Among High-Risk Vietnamese Americans. Am J Public Health. 107: 433-440. 